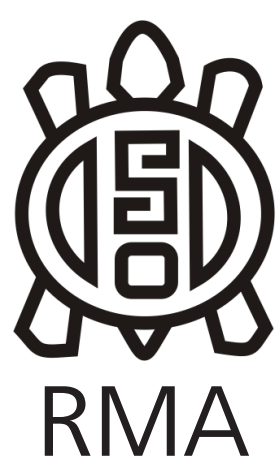

Dossier

\title{
Estudios geoarqueológicos de fuentes y canteras líticas de las Sierras Pampeanas y llanuras adyacentes
}

\author{
Geoarchaeological studies of sources and lithic quarries in the \\ Pampean Sierras and adjacent plains
}

Guillermo Heider*, Ariel Ortiz Suarez*, Diego Rivero**, Edgardo Baldo***,
Sebastián Pastor ${ }^{* * * *}$, Gabriel Ramos*, Mariangeles Borgo ${ }^{* * * * *}$,
Raúl Gil ${ }^{* * * * *}$, Jorge Chiesa*, Carlos Costa*, Andrea Recalde**,
Rafael Curtoni******, Julieta Capriolo* y Lucas Muñoz*

*Departamento de Geología, Facultad de Ciencias Físico, Matemáticas y Naturales,

Universidad Nacional de San Luis. E-mail: guillermoheider@hotmail.com; aortiz@unsl.edu.ar; gramos@unsl.edu.ar; jchiesa@unsl.edu.ar; costa@unsl.edu.ar **IEH-CONICET. Universidad Nacional de Córdoba. E-mail: ayampitin1@yahoo.com.ar; recaldema@yahoo.com.ar,

${ }^{* * * C I C T E R R A, ~ C O N I C E T . ~ U n i v e r s i d a d ~ N a c i o n a l ~ d e ~ C o ́ r d o b a . ~ E-m a i l: ~ e b a l d o @ c o m . u n c o r . e d u ~}$ **** CITCA-CONICET. E-mail: pastorvcp@yahoo.com.ar

*****CONICET-CCT San Luis. Departamento de Geología, Facultad de Ciencias Físico,

Matemáticas y Naturales, Universidad Nacional de San Luis. IFDC-San Luis. E-mail: mariangeles_Borgo@hotmail.com;julietacapriolo@gmail.com;lucasneoclasico@gmail.com

$* * * * * *$ INQUISAL-CONICET. Universidad Nacional de San Luis. E-mail: ragil@unsl.edu.ar

$* * * * * *$ INCUAPA-CONICET. Facultad de Ciencias Sociales, Universidad Nacional de Centro de la Provincia de Buenos Aires. E-mail: rcurtoni@soc.unicen.edu.ar

\begin{abstract}
Resumen
El material lítico es el elemento de mayor abundancia en los registros arqueológicos de Sierras Centrales y sus llanuras adyacentes. Los estudios realizados sobre el mismo utilizan diferentes escalas espaciales, metodologías de campo y laboratorio. Sin embargo, los programas de investigación orientados a la detección de fuentes de aprovisionamiento y canteras arqueológicas no tienen en la región un desarrollo similar al de otras regiones del Argentina. En este trabajo se presentan las líneas iniciales de un proyecto de escala macrorregional, específicamente orientado a su estudio. Los resultados alcanzados hasta el momento permitieron identificar numerosas canteras y fuentes en las provincias de San Luis, Córdoba, La Rioja y Catamarca. En ese marco, proponemos un modelo de yacencia de rocas silíceas. El mismo permite entender por un lado la génesis de las rocas identificadas y, por otra parte, se constituye como el primer modelo predictivo de escala amplia para el centro de Argentina.
\end{abstract}

Palabras Clave: rocas silíceas; sierras y llanuras pampeanas; modelo de yacencia; predictibilidad.

\begin{abstract}
Lithic materials are among the most abundant items in the archaeological record of the Central Ranges and their adjacent plains. The studies carried out with lithic artefacts use different spatial scales, as well as field and laboratory methodologies. However, the research programs oriented to the detection of lithic sources and archaeological quarries do not have in this region a similar development in comparison to other regions of Argentina. This paper presents the initial lines of a macroregional scale project, specifically oriented to their study. The results achieved so far allowed the identification of numerous quarries and lithic sources in San Luis, Córdoba, La Rioja, and Catamarca provinces. We propose a model of deposit of the siliceous rocks which allows to understand the genesis of the identified rocks. On the other hand, it is the first wide-scale predictive occurrence model for the center of Argentina.
\end{abstract}

Keywords: Siliceous rocks; Pampean mountain ranges and plains; occurrence model; predictability model. 
Los estudios multidisciplinarios sobre materias primas líticas en la región arqueológica de Sierras Centrales (González 1952) y sus llanuras adyacentes se han incrementado de manera notoria (Heider et al. 2017; Sario et al. 2017; Cattáneo et al. 2018; entre otros). Sin embargo, los mismos se enmarcan en miradas restringidas en términos espaciales, así como en una escasez relativa de estudios previos. Cuando se realizaron abordajes sistemáticos, de manera general, se orientaron a resolver cuestiones locales y/o regionales (véase Heider et al. 2015 y trabajos allí citados). Más aún, la mayoría se concentró en la caracterización petrográfica del recurso lítico y en un análisis tecnológico de los materiales arqueológicos. A partir de esos antecedentes es que se presentan los primeros resultados de un proyecto multidisciplinario de escala macrorregional (figura 1). El mismo abarca regiones llanas y semiáridas de las provincias de San Luis, La Rioja y

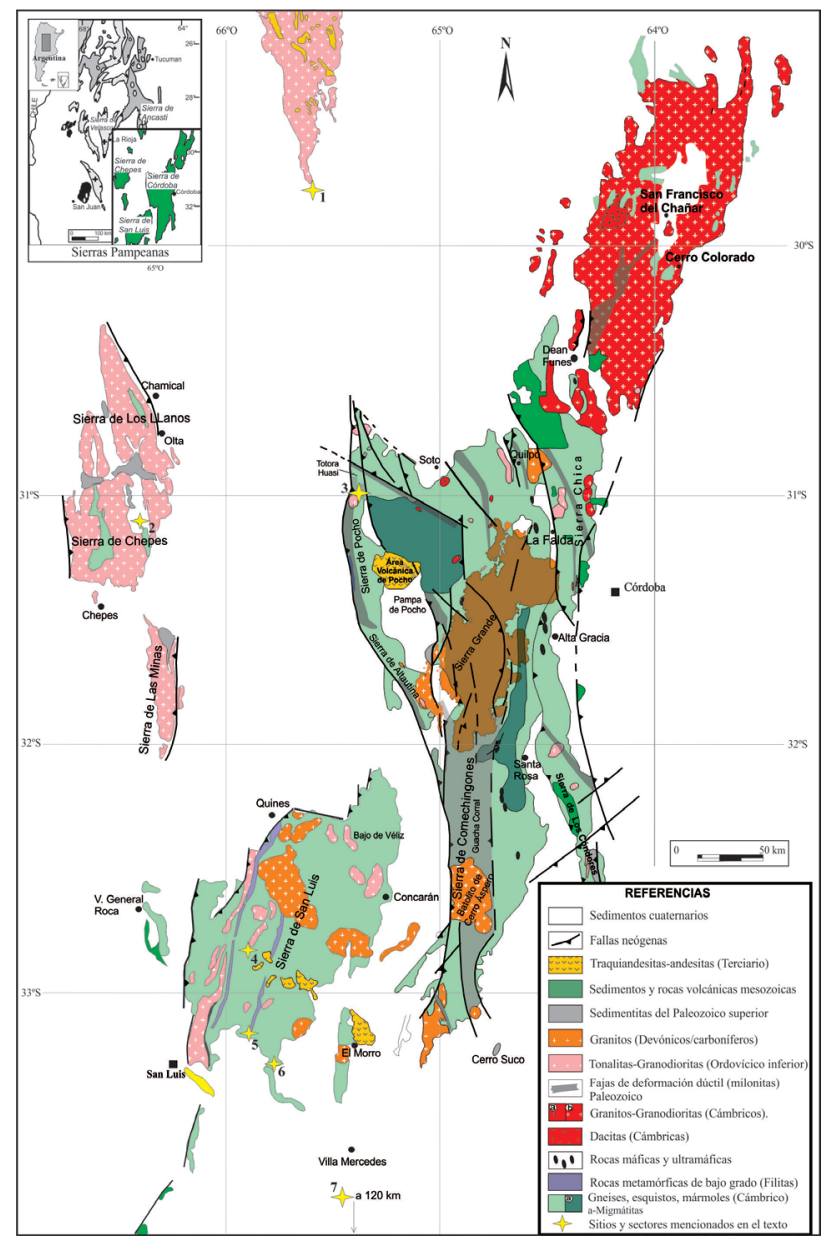

Figura 1: Mapa geológico macroregional. Elementos mencionados en el texto: (1) Loma de Los PedernalesCatamarca, (2) Los Barrancos, (3) Mina Clavero, (4) Laguna Brava, (5) Cuenca media del río Quinto, (6) El Lechuzo, (7) Loma de los Perdernales-San Luis.

Figure 1: Macroregional geological map. Elements mentioned in the text: (1) Loma de Los Pedernales-Catamarca, (2) Los Barrancos, (3) Mina Clavero, (4) Laguna Brava, (5) Middle basin of the Quinto River, (6) El Lechuzo, (7) Loma de los Perdernales-San Luis.
Catamarca, así como diferentes áreas de la Sierras de San Luis, Sierras de Los Llanos (La Rioja), Sierras Grandes de Comechingones, Sierras Chicas y Sierras del Norte (las tres últimas en la provincia de Córdoba). En su etapa inicial, el proyecto tiene tres objetivos generales: a) detectar fuentes de materia prima y canteras arqueológicas; b) caracterizar ambas a nivel tecno-tipológico, petrográfico y geoquímicos; y c) analizar que procesos geológicos y geomorfológicos intervinieron en su génesis y transformación. Este último es el abordado aquí, para lo cual proponemos un primer modelo de yacencia de rocas silíceas de uso arqueológico para el centro de Argentina.

\section{Material y métodos}

Esta publicación incluye ámbitos geológicos disimiles. La mayor parte queda incluida en las Sierras Pampeanas Orientales (Caminos 1979), las cuales comparten un mismo estilo morfológico y tectónico caracterizado por bloques de rocas cristalinas (ígneas y metamórficas), limitados por fallas inversas de alto ángulo que definen una serie de valles de dirección norte-sur (González Bonorino 1950). Los espacios extra-serranos son depósitos eólicos del Mar de Arena Pampeano (Iriondo 1999) en el sector sur e interestratificados aluvialeseólicos con horizontes fluviales y paleosuelos en el centro y norte (Irigoyen 1981, Limarino et al. 1999). De modo similar quedan incluidas en su interior las regiones arqueológicas de Sierras Centrales, Los Llanos de La Rioja y el norte de Pampa Seca (González 1952; Politis y Barros 2003-2004). En ese contexto, los estudios de fuentes y canteras son recientes en relación a otras líneas de investigación desarrolladas por los integrantes del equipo (i.e. agricultura, arte rupestre, estudios regionales o poblamiento temprano). Su análisis implica metodologías similares en todas las áreas para, de ese modo, obtener resultados comparables. Principalmente técnicas surgidas desde la arqueología distribucional (sensu Ebert 1992), la geoarqueología (sensu Waters 1992) y estudios tecno-tipológicos clásicos (Aschero 1975-1983; Aschero y Hocsman 2004). Los estudios de laboratorio se basan en cortes petrográficos con luz natural y polarizada, DRX y espectrometría de masas, utilizadas como etapas complementarias de trabajo (Heider y Demichelis 2015; Heider et al. 2017).

Los materiales identificados corresponden a siete canteras arqueológicas y seis fuentes de rocas silíceas sin rastros de usos antrópicos. En la Tabla 1 se observan los nombres de las mismas, así como su ubicación y el tipo de estudio ya realizado. En el caso de la cuenca del río Quinto, la información fue visualmente resumida dividiendo a la misma en subsectores. En la Cuenca superior se identificaron dos canteras (El Chiquero y La Falla), y tres fuentes (Laguna Brava, Cuatro Esquinas y La Petra). Por su parte, en la Cuenca media se registraron tres fuentes de calcedonia: Paso de Las Carretas, Río Quinto y Paso de la Caballada. Todas las fuentes identificadas son primarias, 


\begin{tabular}{|c|c|c|c|c|c|c|c|}
\hline Canteras & Fuentes & $C-P$ & DRX & $\mathrm{E}-\mathrm{M}$ & $\mathrm{E}-\mathrm{T}$. & A-D. & GEO \\
\hline Loma de los Pedernales (CT) & & $\mathrm{X}$ & $\mathrm{X}$ & & $\mathrm{X}$ & $\mathrm{X}$ & $\mathrm{X}$ \\
\hline Los Barrancos (LR) & & $X$ & & & & $\mathrm{X}$ & $X$ \\
\hline Mina Clavero (CBA) & & $X$ & $X$ & & $X$ & & \\
\hline Alto El Lechuzo (SL) & & $\mathrm{X}$ & & $x$ & $x$ & $x$ & $x$ \\
\hline \multirow[t]{2}{*}{ Loma de Los Pedernales (SL) } & & $\mathrm{X}$ & & $x$ & $x$ & $x$ & $x$ \\
\hline & Laguna Brava (SL) & $X$ & & & $X$ & $x$ & $x$ \\
\hline \multirow[t]{3}{*}{ El Chiquero (SL) } & & $X$ & & & $x$ & $x$ & $x$ \\
\hline & Cuatro Esquinas (SL) & $X$ & & & $X$ & $X$ & $x$ \\
\hline & La Petra (SL) & $X$ & & & $\mathrm{X}$ & $x$ & $\mathrm{X}$ \\
\hline \multirow[t]{4}{*}{ La Falla (SL) } & & $\mathrm{X}$ & & & $X$ & $\mathrm{X}$ & $X$ \\
\hline & Paso de las Carretas (SL) & $X$ & & & $X$ & $x$ & $x$ \\
\hline & rio Quinto $(\mathrm{SL})$ & $\mathrm{X}$ & & & $x$ & $x$ & $\mathrm{X}$ \\
\hline & Paso de la Caballada (SL) & $\mathrm{X}$ & & & $\mathrm{X}$ & $\mathrm{X}$ & $x$ \\
\hline
\end{tabular}

Tabla 1: Fuentes de materia prima y canteras arqueológicas estudiadas por el proyecto. Glosario: CT (Catamarca), LR (La Rioja), (CBA) Córdoba, SL (San Luis), C-P (cortes petrográficos), DRX (difractometría de rayos X), E-M (espectrometría de masas), E-T (estudios tecno-tipológicos), A-D (análisis distribucional), GEO (estudios geológicos in situ).

Table 1: Sources of lithic raw material and archaeological quarries studied by the project. Glossary: CT (Catamarca), LR (La Rioja), (CBA) Córdoba, SL (San Luis), C-P (petrographic cuts), XRD (X-ray diffractometry), E-M (mass spectrometry), E-T (technotypological studies), A-D (distributional analysis), GEO (geological in situ studies).

con excepción de La Petra (sensu Nami 1992). Si bien todas fueron presentadas durante el Congreso Argentino de Estudios Líticos en Arqueología (Heider et al. 2018), solo algunas cuentan con estudios previos publicados en revistas y congresos (Heider et al. 2017; Borgo et al. 2018, entre otros).

\section{Resultados}

Un conjunto de estudios mencionados en el acápite inicial enumera las escasas caracterizaciones sobre rocas silíceas realizados en el centro de Argentina con anterioridad a este proyecto. En este sentido, se ha logrado un notorio aumento de sitios en proporción al período de tiempo que lleva el proyecto en desarrollo. En línea con los objetivos planteados para este trabajo, la presentación de los resultados se centrará casi de modo exclusivo enlas materias primas identificadas y su asociación con los ambientes geológicos circundantes. Esa relación es, en definitiva, la base empírica a partir de la cual proponemos un primer "modelo de yacencia de rocas silíceas para el centro de Argentina".

En posición septentrional, entre las canteras identificadas, se encuentra Loma de los Pedernales (Catamarca). Los materiales arqueológicos fueron recuperados en una superficie de 9 hectáreas que incluyó, al menos, 27 montículos de escombros en donde se ve representada toda la secuencia técnica de producción de artefactos. Geológicamente es una lomada baja compuesta por sedimentos neógenos de la Fm. Los Llanos (Ezpeleta et al. 2006), dentro de los cuales fueron identificadas las calcedonias usadas por los pueblos originarios. Al sur de esta cantera, dentro de sierra de Chepes-Los Llanos se encuentra la cantera Los Barrancos (La Rioja). El material de uso arqueológico es una calcedonia roja, identificada frecuentemente en puntas de proyectil triangulares pequeñas del Holoceno tardío final (Heider et al. 2018). Los estudios realizados muestran que las actividades iniciales de extracción implicaron la ruptura de una roca de caja dentro de la cual se incluye la calcedonia en forma de venillas de espesores máximos de $2 \mathrm{~cm}$. Creemos que el material fue trasladado, para finalizar la secuencia tecnológica en sitios cercanos donde el arte rupestre se destaca como referente de las prácticas sociales. Geológicamente las venillas de calcedonia están dentro de un dique aplítico, intrusivos a esquistos y se ubica a aproximadamente $80 \mathrm{~m}$ del contacto de un cuerpo granítico, denominado granodioriota de Chepes (Dahlquist et al. 2005). En el sudoeste de Córdoba se identificó la cantera Mina Clavero (Córdoba). La calcedonia de color blanco fue recuperada en depósitos secundarios a lo largo de la costa del río de Los Sauces. Geológicamente, la fuente primaria (actualmente sin evidencias de canteo) son depósitos discontinuos ubicados en las barrancas próximas, e insertos en los depósitos neógenos de la Fm. Brochero (Sayago 1979; Heider et al. 2015, 2018). La Falla es un área de canteras de calcedonia y se describe en detalle en este mismo dossier (Borgo et al. 2018). Las dos canteras restantes, Alto El Lechuzo y Loma de Los Pedernales, son depósitos secundarios ubicados en espacios de la llanura (Heider et al. 2015; Heider et al. 2017). En un ambiente geológico sedimentario, el material disperso en lomadas bajas procede de carbonatos posibles de correlacionar con la Fm. Paso de Las Carretas.

Las fuentes sin evidencias de uso antrópico identificas en la cuenca alta y media del río Quinto tienen dos ambientes geológicos diferentes. Laguna Brava es un afloramiento de ópalo amarillo solo observable, hasta el momento, en los destapes de una cantera sub-actual de 
cuarzo. Geológicamente se corresponde con las porciones terminales de la pegmatita San Luis I (Llambías et al. 1991). El resto de las fuentes (véase acápite anterior), se corresponden con sílices tipo calcedonia (Heider et al. 2017), propias de un ambiente geológico sedimentario (neógeno), específicamente la Formación Paso de las Carretas (Basaez y Chiesa 2014).

\section{Discusión y consideraciones finales}

El cuarzo puede ser postulado como la roca de mayor presencia en los sitios arqueológicos del centro de Argentina (Heider et al. 2015; Cattáneo et al. 2018). Su abundancia en numerosos registros, así como su alta disponibilidad en muchos sectores del paisaje, limita, hasta el momento, su utilización para estudiar aspectos como la movilidad, la interacción y la estacionalidad de las ocupaciones humanas (Cattáneo et al. 2018). Las rocas silíceas son, en ese sentido, mejores trazadores de esas problemáticas a través del tiempo. El proyecto

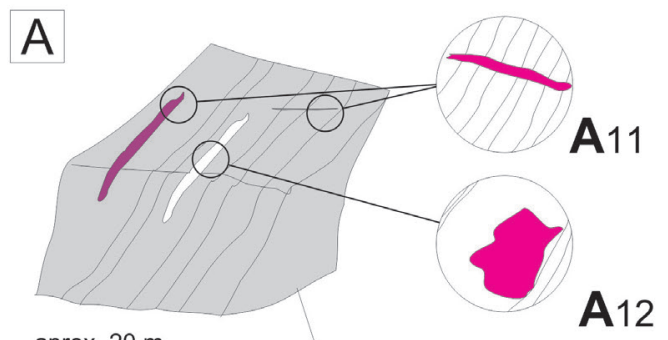

aprox. $20 \mathrm{~m}$

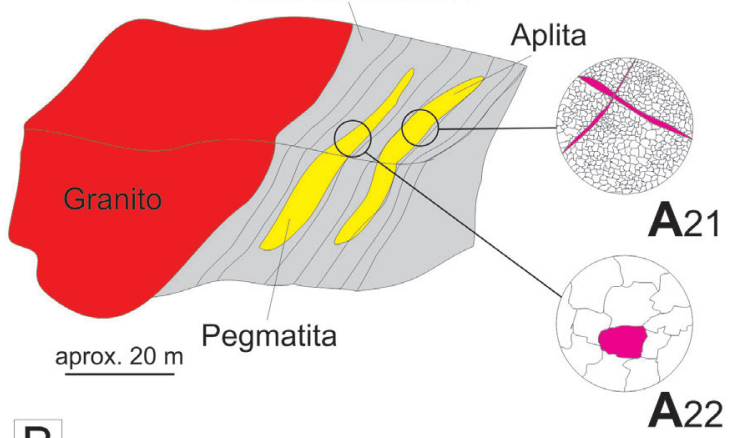

B

A22

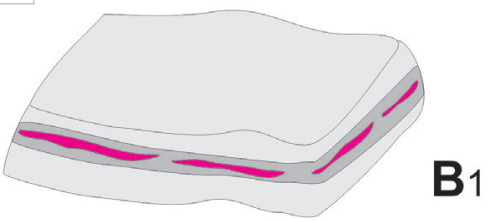

$\underline{\text { aprox. } 1 \mathrm{~m}}$

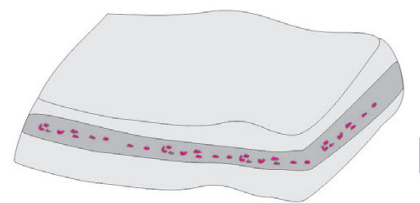

B2

Figura 2: Modelo de yacencia de rocas silíceas para el centro de Argentina

Figure 2: Siliceous rock lying model for central Argentina en desarrollo, expuesto solo parcialmente aquí, intenta caracterizar desde diferentes aspectos esas materias primas en un continuum que va desde las canteras a los sitios. Los trabajos de campo permitieron identificar más de una decena de elementos del paisaje en donde aflora o se depositan estas rocas. Los estudios geoarqueológicos, por su parte, se orientaron a su clasificación a nivel petrográfico y geoquímico. Con esa base, y apoyados por estudios multiproxy en el campo, se desarrolló un "Modelo de yacencia de rocas silíceas para el centro de Argentina" (Figura 2).

Los modos de yacencia se pueden ordenar en dos grupos primarios principales (Figura 2): A) cuarzo y calcedonia en rocas del basamento ígneo-metamórfico; B) calcedonia y ópalos en sedimentos cenozoicos (Figura 3). En el primer caso, identificamos subgrupos: En $\left(A_{1}\right)$ las fuentes y canteras se ubicarían en rocas metamórficas constituyendo filones de cuarzo con dimensiones decimétricas o calcedonia en cuerpos irregulares de tamaños no superiores a $1 \mathrm{~m}\left(\mathrm{~A}_{11}\right.$ [El Chiquero, Borgo et al. 2017]) o en venas discordantes o pequeños diques que cortan la foliación metamórfica de la roca de caja ( $A_{12}$ [La Suiza -Sario 2013]). También en este grupo se reconoce calcedonia relacionada a granitos $\left(A_{2}\right)$, ya sea calcedonia en aplitas ( $A_{21}$ [Los Barrancos, este trabajo]), o cuarzo-calcedonia en cuerpos pegmatíticos ( $A_{22}$ [La Falla, Borgo et al. 2017]). Por otra parte, las calcedonias que alojan en depósitos sedimentarios cenozoicos ricos en carbonatos, probablemente de edad neógena (B), se pueden encontrar en bancos concordantes con la

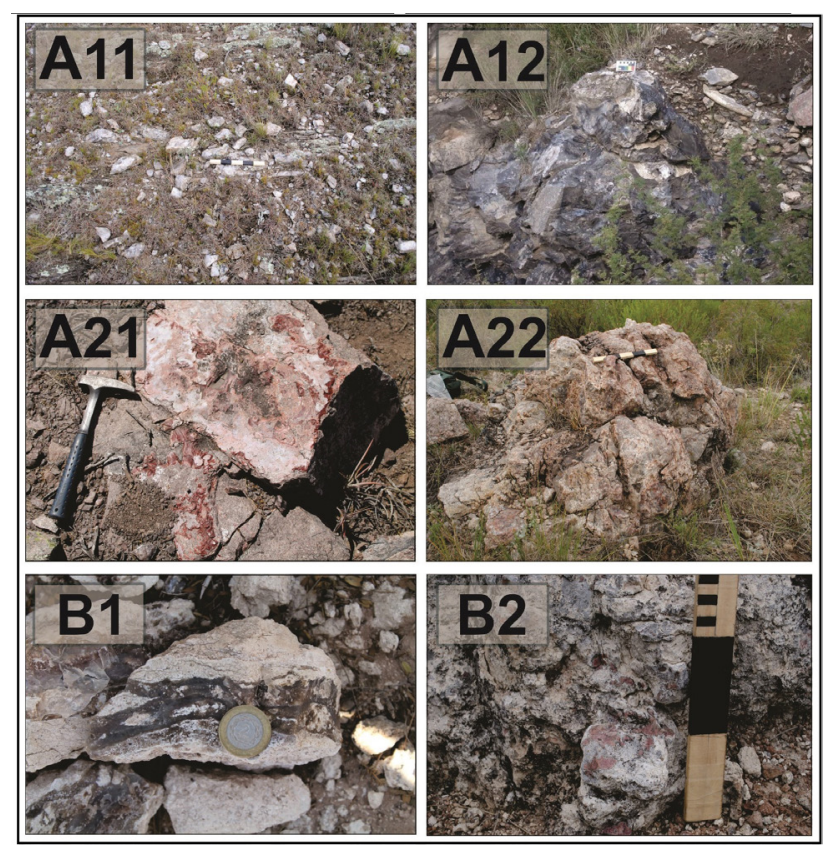

Figura 3: Imágenes de los modos de yacencia identificados en el terreno

Figure 3: Images of the yacencia modes identified in the field. 
estratificación horizontal o subhorizontal, con espesores superiores al metro ( $B_{1}$ [Lomas de Los Pedernales (ambas), Alto del Lechuzo, Mina Clavero, Heider et al. 2017]) o en pequeñas concreciones que forman agregados irregulares ( $B_{2}$ [Paso de Las Carretas, Borgo et al. 2017]).

Los modos de yacencia sugieren distintos orígenes, el grupo A se relaciona a la evolución del basamento de las Sierras Pampeanas, ocurrida fundamentalmente en el Paleozoico, que ha dado lugar a la migración de fluidos silíceos vinculada a procesos magmáticos y metamórficos en el interior de la corteza terrestre. El grupo B, en cambio, representa materiales más jóvenes, formados por la precipitación de fluidos en ambientes más superficiales, probablemente controlada por la composición química del sedimento hospedante. Se espera que la caracterización química y petrográfica de los materiales que actualmente se desarrolla permita reconocer la proveniencia cuando se encuentren fuera de su lugar de origen. Finalmente, proponemos este modelo como una herramienta perfectible que oriente la búsqueda desde el comienzo mismo de las tareas de investigación (búsqueda bibliográfica y prospecciones) y acompañe explicaciones sobre la génesis geológica de los elementos identificados.

San Luis, 25 de febrero de 2018

\section{Agradecimientos}

Queremos agradecer a los pueblos originarios de La Rioja, Catamarca, San Luis y Córdoba. A los gobiernos provinciales por los permisos y el apoyo. Al personal técnico de los distintos laboratorios que realizaron las tareas de procesamiento y análisis inicial de datos. A nuestros amigos, que en cada paraje, pueblo o ciudad nos reciben con afecto. Nos financiaron: Secyt-UNC (30720130100910CB); PIP-2017-2019-GI; 18-249-APNMCT; PICT-2013-0290; PROICO-148-2018-2020-UNSL. Las sugerencias de los evaluadores mejoraron este trabajo, en el que todo lo expuesto es responsabilidad de los autores.

\section{Referencias citadas}

Aschero, C. (1975-1983). Ensayo para una clasificación morfológica de artefactos líticos. Informe a CONICET. Ms.

Aschero, C. y Hocsman S. (2004). Revisando cuestiones tipológicas en torno a la clasificación de artefactos bifaciales. Temas de Arqueología. Análisis Lítico: 7-25.

Basaez, A. y Chiesa, J. (2014). Análisis de facies de la Formación Paso de las Carretas (Neógeno, San Luis). Actas del XIX Congreso Geológico Argentino T1: 7. Córdoba, Argentina.

Borgo, M., Heider, G., Ramos G. y Curtoni R. (2017). Primeros avances en el área de canteras La Falla (San Luis).
Congreso Argentino de Estudios Líticos en Arqueología: 32-34. Córdoba.

Caminos, R. (1979). Sierras Pampeanas Noroccidentales. Salta, Tucumán, Catamarca, La Rioja y San Juan. 2 Simposio Geología Regional Argentina (1) 225-291. Córdoba.

Cattaneo, R., Sario, G., Caminoa, J., Collo, G., Rubio, M., Germanier, A., Faudone, S., Izeta, A. y Salvatore, M. (2018). Caracterización química de fuentes de abastecimiento e instrumental arqueológico de cuarzo de la provincia de Córdoba (Argentina). pp 50. VII Jornadas Arqueológicas de Cuyo, Malargüe.

Dahlquist, J., Rapela, C., Baldo, E. (2005). Petrogenesis of cordierite-bearing S-type granitoides in Sierra de Chepes, Famatinian orogen, Argentina. Journal of South American Earth Sciences, 20: 231-251

Ebert, J. (1992), Distributional Archaeology. University of New Mexico Press, New Mexico.

Ezpeleta, M., Dávila, F. y Astini, R. (2006). Estratigrafía y paleoambientes de la Formación Los Llanos (La Rioja): una secuencia condensada Miocena en el antepaís fragmentado andino central. Revista de la Asociación Geológica Argentina 61 (2): 171-186.

González, A. (1952). Antiguo horizonte precerámico en las Sierras Centrales, Argentina. Runa V: 110-133.

González Bonorino, F. (1950). Algunos problemas geológicos de Sierras Pampeanas. Revista de la Asociación Geológica Argentina 5 (3): 81-110

Heider, G y Demichelis A. (2015). Loma de Los Pedernales, a local raw material source in the North of Pampa Seca, Argentina. Quaternary International 375: 3-12.

Heider G., Rivero, D. y Baldo, E. (2015). Rocas de uso arqueológico en Sierras Centrales. Fuentes de recursos líticos identificadas y potenciales en las provincias de Córdoba y San Luis, Argentina. Revista de Antropología del Museo de Entre Ríos 1 (2): 55-72.

Heider G., Ortiz Suarez, A., Gil, R., Perino, E., Chiesa, J., Basaez, A., Bazán C., y Díaz I. (2017). Chert's source in Las Travesías from the center of Argentina. A case study in the arid zone. 11th International Symposium on knappable material, "From toolstone to stone tools".156-157. Buenos Aires y Necochea, Argentina.

Irigoyen, M. (1981). Geología y recursos naturales de San Luis. VIII Congreso Geológico Argentino. Buenos Aires.

Iriondo, M. (1990). A Late Holocene dry period in the Argentine Plains. Quaternary of South America and 
Llambías, E, Caminos, R., Cingolani, C., Prozzi C., Ortíz Suarez, Saavedra A. y Toselli, J. (1991). Las granodioritas sincinemáticas de la Sierra de San Luis. $6^{\circ}$ Congreso Geológico Chileno: 187-191.

Limarino, O., Poma, S. y Miró, R. (1999). Hoja Geológica Chamical 3166 - I. Provincias de La Rioja, San Luis y San Juan. Programa Nacional de Cartas Geológicas 1:250.000. SEGEMAR.

Nami, H. (1992). El subsistema tecnológico de la confección de instrumentos líticos y la explotación de los recursos del ambiente: una nueva vía de aproximación. Shincal 2: 33-53.

Politis, G. y Barros P. (2003-2004). La región Pampeana como unidad espacial de análisis en la arqueología
Sario, G. (2013). Sources of lithic material procurement in Estancia La Suiza archeological locality (San Luis, Argentina). Journal of Archaeological and Anthropological Sciences 5(3): 245-254.

Sario, G, Pautassi E. y Salvatore M. (2017). Canterastaller El Ranchito (Dpto. Ischilín, Córdoba). Una primera aproximación en la caracterización de fuentes y al análisis de los conjuntos líticos. Revista del Museo de Antropología, Suplemento especial 1: 59-64

Sayago, J. (1979). Geomorfología del Valle de San Alberto. Provincia de Córdoba. $7^{\circ}$ Congreso Geológico Argentino 2: 89-107.

Waters, R. (1992). Principles of Geoarchaeology. The University of Arizona Press, Tucson. 